\title{
Differentiability with respect to parameters of average values in probabilistic contracting dynamical systems $\dagger$
}

\author{
W. DOUGLAS WITHERS \\ Department of Mathematics, U.S. Naval Academy, Annapolis, Maryland 21402, USA
}

(Received 17 May 1986 and revised 15 May 1989)

Abstract. We consider a dynamical system consisting of a compact subset of $\mathbf{R}^{N}$ or $\mathbf{C}^{N}$ with several contracting maps chosen with prescribed probabilities, which may depend on position. We show that if the maps and the probabilities are $C^{1+\alpha}$ functions of the spatial variable and an external parameter, then the average value of a $C^{1+\alpha}$ function is a differentiable function of the parameter. One implication of this theorem is that for certain families of complex functions dependent on a parameter the reciprocal of the dimension of an invariant measure on the Julia set is a harmonic function of the parameter.

\section{Introduction}

In this article we consider a dynamical system consisting of a subset $X$ of $\mathbf{R}^{N}$ or $C^{N}, n$ contracting functions $\beta_{1}, \ldots, \beta_{n}: X \rightarrow X$, and $n$ continuous positive probabilities $p_{1}, \ldots, p_{n}: X \rightarrow(0,1)$, so that if $x_{k}$ represents the state of the system at time $k$, then $x_{k+1}=\beta_{i}\left(x_{k}\right)$ with probability $p_{i}\left(x_{k}\right)$. The study of this type of system is facilitated by the existence of a unique invariant probability measure $\mu$, which gives long-term trajectory averages:

$$
\lim _{K \rightarrow x} \frac{1}{N} \sum_{k=1}^{N} f\left(x_{k}\right)=\langle\mu, f\rangle,
$$

as is proved in $\S 2$. This type of system has been studied by Karlin [8], Barnsley \& Demko [1], Barnsley \& Elton [3], Barnsley et al. [2], Barnsley \& Harrington [4], and Elton [7]. The equilibrium measures on Julia sets studied by Brolin [5] are also an example of such an invariant measure.

We suppose further that the system is dependent on an external parameter $w \in W$, where $W$ is a subset of $\mathbf{R}^{N}$ or $\mathbf{C}^{N}$, so that in fact $\beta_{1}, \ldots, \beta_{n}: X \times W \rightarrow X$ and $p_{1}, \ldots, p_{n}: X \times W \rightarrow(0,1)$. Concerning this type of system we prove the following theorem:

THEOREM. If $\beta_{1}, \ldots, \beta_{n} ; p_{1}, \ldots, p_{n}$; and $f$ are $C^{1+a}$ functions, then $\langle\mu, f\rangle$ is $a$ differentiable function of the parameter $w$.

† This research was supported by a grant from the Naval Academy Research Council. 
The structure of this article is as follows. In $\S 2$ we prove the existence and uniqueness of $\mu$. In $\S 3$ we prove our main theorem that under appropriate hypotheses $\langle\mu, f\rangle$ is a differentiable function of the parameter $w$. In $\S 4$ we apply our results to the study of invariant measures on Julia sets, obtaining the result that for certain families of complex functions, including the functions $F(z)=z^{2}-w$, for values of $w$ outside the Mandelbrot set, the reciprocal of the dimension of the invariant measure on the Julia set is a harmonic function of the parameter.

\section{The unique invariant probability measure}

In this section we show that a system of the type described in the Introduction with positive Hölder-continuous probabilities has a unique invariant probability measure. Throughout this section we make the following assumptions:

(i) $(X, \rho)$ is a compact metric space.

(ii) The functions $\beta_{1}, \ldots, \beta_{n}$ from $X$ to $X$ are contractions; i.e., there exists $\lambda<1$ such that

$$
\rho\left(\beta_{i}(x), \beta_{i}(y)\right) \leq \lambda \rho(x, y) .
$$

(iii) The functions $p_{1}, \ldots, p_{n}$ from $X$ to $(0, \infty)$ are probabilities; $\sum p_{i}(x) \equiv 1$.

(iv) The functions $p_{i}$ are Hölder continuous and positive and thus bounded away from zero; there exists $q>0$ such that $p_{i}(x) \geq q$ for all $x$. For convenience we assume $q<\frac{1}{2}$.

Let $C$ be the space of real-valued continuous functions on $X$; then its dual space $C^{*}$ is the space of finite Borel measures on $X$; an element $m$ of $C^{*}$ is a probability measure if and only if $\langle m, f\rangle \geq 0$ for all $f \geq 0$ and $\langle m, h\rangle=h$ for all constant functions $h$. Let $T: C \rightarrow C$ be defined by

$$
(T f)(x)=\sum_{i} p_{i}(x) f\left(\beta_{i}(x)\right)
$$

Then the adjoint operator $T^{*}$ of $T$ is a bounded linear operator from $C^{*}$ to $C^{*}$. If a measure $\mu$ represents trajectory averages as in equation (1), then $\mu$ must be a probability measure and $\mu$ must be a fixed point of $T^{*}$. We show that these conditions make $\mu$ unique, and for any probability measure $m \in C^{*}$, the sequence $T^{* k} m$ converges weakly to $\mu$.

We use the symbol $\Gamma(f)$ to denote $\sup f-\inf f$. Note that $\Gamma$ is a seminorm on $C$.

LEMMA 2.1. Let $\delta$ be a modulus of continuity for $f$ :

$$
|f(x)-f(y)| \leq \delta(\rho(x, y)) \text {. }
$$

Then

$$
\Gamma\left(T^{k} f\right) \leq\left(1-2 q^{k}\right) \Gamma(f)+2 q^{k} \delta\left(\lambda^{k} \operatorname{diam} X\right) .
$$

Proof. If $\mathbf{i}$ denotes the $k$-tuple $\left(i_{1}, \ldots, i_{k}\right)$, then we let

$$
\beta_{i}(x)=\beta_{i_{1}}\left(\beta_{i_{2}}\left(\cdots \beta_{i_{k}}(x)\right)\right)
$$

and

$$
p_{\mathbf{i}}(x)=\prod_{j=1}^{k} p_{i_{i}}\left(\beta_{i_{i+1}}\left(\cdots \beta_{i_{k}}(x)\right)\right)
$$


Then we may write

$$
\left(T^{k} f\right)(x)=\sum_{\mathbf{i}} p_{\mathbf{i}}(x) f\left(\beta_{\mathbf{i}}(x)\right) .
$$

Note that the functions $\beta_{\mathrm{i}}$ and $p_{\mathrm{i}}$ satisfy assumptions (ii), (iii), and (iv) with different constants;

$$
\rho\left(\beta_{i}(x), \beta_{i}(y)\right) \leq \lambda^{k} \rho(x, y) ;
$$

$\sum p_{\mathrm{i}}(x) \equiv 1$; and $p_{\mathrm{i}}(x) \geq q^{k}$ for all $x$.

Let $X_{k}$ denote the set $\bigcup \beta_{\mathrm{i}}(X)$, where the union is taken over all $k$-tuples $\mathbf{i}$. Then there exists $y \in X$ and a $k$-tuple $\mathbf{j}$ such that $f\left(\beta_{j}(y)\right)=\inf _{X_{k}} f$, and for any $x \in X$ we have

$$
f\left(\beta_{\mathrm{j}}(x)\right) \leq \inf _{X_{k}} f+\delta\left(\lambda^{k} \operatorname{diam} X\right) .
$$

We have

$$
\begin{aligned}
T^{k} f(x) & =\sum_{\mathbf{i}} p_{\mathrm{i}}(x) f\left(\beta_{\mathrm{i}}(x)\right) \\
& =\sum_{\mathbf{i} \neq \mathbf{j}} p_{\mathrm{i}}(x) f\left(\beta_{i}(x)\right)+p_{\mathrm{j}}(x) f\left(\beta_{j}(x)\right) \\
& \leq\left(1-p_{\mathrm{j}}(x)\right) \sup _{x_{k}} f+p_{\mathrm{j}}(x)\left(\inf _{X_{k}} f+\delta\left(\lambda^{k} \operatorname{diam} X\right)\right) \\
& \leq \sup _{x_{k}} f+p_{\mathrm{j}}(x)\left(\inf _{X_{k}} f+\delta\left(\lambda^{k} \operatorname{diam} X\right)-\sup _{X_{k}} f\right) \\
& \leq \sup _{X_{k}} f+q^{k}\left(\inf _{X_{k}} f+\delta\left(\lambda^{k} \operatorname{diam} X\right)-\sup _{X_{k}} f\right) .
\end{aligned}
$$

Similarly, we may obtain

$$
T^{k} f(x) \geq \inf _{X_{k}} f+q^{k}\left(\sup _{X_{k}} f-\delta\left(\lambda^{k} \operatorname{diam} X\right)-\inf _{X_{k}} f\right) .
$$

Substracting the two inequalities, and noting that

$$
\sup _{x_{k}} f-\inf _{x_{k}} f \leq \Gamma(f),
$$

we obtain

$$
\Gamma\left(T^{k} f\right) \leq\left(1-2 q^{k}\right) \Gamma(f)+2 q^{k} \delta\left(\lambda^{k} \operatorname{diam} X\right) .
$$

THEOREM 2.2. There exists a unique probability measure $\mu$ invariant under $T^{*}$. Moreover, for any $m \in C^{*}$ with $\langle m, 1\rangle=1$, the sequence of measures $T^{* k} m$ converges weakly to $\mu$.

Proof. First we show that the sequence of functions $T^{k} f$ converges in $C$ to a constant. It is not difficult to show that the sequence sup $T^{k} f$ is nonincreasing and the sequence inf $T^{k} f$ is nondecreasing. We show that the sequence $\Gamma\left(T^{k} f\right)$ decreases to zero by finding a subsequence which decreases to zero.

Using the Hölder continuity of the functions $p_{i}$ it can be shown that the family of functions $T^{k} f$ is uniformly equicontinuous. Thus we can choose a modulus of continuity $\delta$ for $T^{k} f$ independently of $k$. We also choose $\delta$ so that it has an inverse function and $\sup \delta=\Gamma(f)$. 
We define our subsequence iteratively: let $a_{0}=\Gamma(f)$. When $a_{0}, \ldots, a_{1}$, have been defined, we define $a_{l+1}$ as follows. Let $j$ be the integer with $a_{l}=\Gamma\left(T^{j} f\right)$. Choose an integer $k$ so that

$$
\frac{1}{\log \lambda} \log \left(\frac{\delta^{-1}\left(a_{1} / 2\right)}{\operatorname{diam} X}\right)<k \leq \frac{1}{\log \lambda} \log \left(\frac{\delta^{-1}\left(a_{l} / 2\right)}{\operatorname{diam} X}\right)+1 .
$$

It can be verified that $k$ is nonnegative. We set $a_{1+1}=\Gamma\left(T^{j+k}(f)\right)$. With this choice of $k$, we have

$$
\delta\left(\lambda^{k} \operatorname{diam} X\right) \leq a_{l} / 2
$$

Thus, from the preceding lemma,

$$
\begin{aligned}
a_{l+1}=\Gamma\left(T^{k}\left(T^{j}(f)\right)\right. & \leq\left(1-2 q^{k}\right) \Gamma\left(T^{j} f\right)+2 q^{k}\left(a_{l} / 2\right) \\
& \leq\left(1-2 q^{k}\right) a_{l}+q^{k} a_{l}=\left(1-q^{k}\right) a_{l} .
\end{aligned}
$$

On the other hand, from the upper bound on $k$, we can obtain:

$$
q^{k} \geq q\left(\frac{\delta^{-1}\left(a_{l} / 2\right)}{\operatorname{diam} X}\right)^{\log q / \log \lambda} .
$$

Thus

$$
a_{l+1} \leq\left(1-q^{k}\right) a_{l} \leq\left(1-q\left(\frac{\delta^{-1}\left(a_{l} / 2\right)}{\operatorname{diam} X}\right)^{\log q / \log \lambda}\right) a_{l} .
$$

Let us define $g:\left(0, a_{0}\right) \rightarrow \mathbf{R}$ by

$$
g(x)=\left(1-q\left(\frac{\delta^{-1}(x / 2)}{\operatorname{diam} X}\right)^{\log q / \log \lambda}\right) x .
$$

Clearly, $g(x) \leq x$; thus $g$ maps $\left(0, a_{0}\right)$ into itself. Moreover, 0 is the unique fixed point of $g$; it follows that $g^{n}\left(a_{0}\right) \rightarrow 0$. Since $a_{l+1} \leq g\left(a_{l}\right) \leq g^{n}\left(a_{0}\right)$, we have $a_{l} \rightarrow 0$ and $\Gamma\left(T^{k} f\right) \rightarrow 0$.

Define $\mu \in C^{*}$ by

$$
\langle\mu, f\rangle=\lim T^{k} f
$$

It is easily shown from the properties of $T$ that $\mu$ is a probability measure and a fixed point for $T^{*}$. Let $m \in C^{*}$. Then

$$
\lim \left\langle m, T^{k} f\right\rangle=\left\langle m, \lim T^{k} f\right\rangle=\langle m,\langle\mu, f\rangle\rangle=\langle m, 1\rangle\langle\mu, f\rangle ;
$$

thus if $\langle m, 1\rangle=1$ then the sequence $T^{* k} m$ converges weakly to $\mu$.

The fact that $\mu$ is a fixed point of the operator $T^{*}$ is equivalent to the invariance property

$$
\mu \beta_{i}(E)=\int_{E} p_{i} d \mu
$$

when the sets $\beta_{i}(E)$ are nonoverlapping.

I am indebted to M. F. Barnsley, J. H. Elton, S. G. Demko, and J. S. Geronimo for pointing out that more than ordinary continuity of the probabilities $p_{i}$ is necessary to prove that the family $\left\{T^{h} f\right\}$ is uniformly equicontinuous. Karlin [8] overlooked this point. It is still an open question whether there is a unique invariant probability 
measure if the probabilities $p_{i}$ are merely continuous. Elton [7] and Barnsley et al. [2] prove the existence of a unique invariant probability measure under hypotheses weaker than those used here, including cases where the functions $\beta_{1}, \ldots, \beta_{n}$ are not always contracting.

The following example shows that the requirement that the functions $p_{i}$ be bounded away from zero cannot be omitted. Let $X=[0,1], \beta_{1}(x)=x / 2, \beta_{2}(x)=(x+1) / 2$, $p_{1}(x)=1-x$, and $p_{2}(x)=x$. Then any measure which assigns weight $\omega$ to the point 0 and weight $1-\omega$ to the point 1 is a fixed point of $T^{*}$.

We note that the theorem of this section remains true if we consider complexvalued functions $f$ and complex-valued measures. This can be shown by application of the previous theorem to the real and imaginary parts of $f$.

\section{The main theorem}

In this section we assume that $X$ and $W$ are compact subsets of $\mathbf{R}^{N}$ with connected interiors. We will use $|\cdot|$ to represent the norms on $X, W$, and $X \times W$. The functions $\beta_{i}$ and $p_{i}$ are dependent on $w \in W$ as well as $x \in X$, so that the average value $\langle\mu, f\rangle$ is a function of $w$.

For differentiable manifolds $Y$ and $Z$ we denote by $C^{\alpha}(Y, Z)$ the space of functions from $Y$ to $Z$ that are Hölder-continuous with exponent $\alpha$. We denote by $C^{1+\alpha}(Y, Z)$ the space of differentiable functions from $Y$ to $Z$ whose derivatives are Hölder continuous with exponent $\alpha$.

TheOREM 3.1. Suppose $\beta_{1}, \ldots, \beta_{n}: X \times W \rightarrow X$ are in $C^{1+\alpha}(X \times W, X)$, and there exists $0<\lambda<1$ such that

$$
\left\|\frac{\partial}{\partial x} \beta_{i}(x, w)\right\|<\lambda
$$

for $i=1, \ldots, n$. Suppose further that the probabilities $p_{1}, \ldots, p_{n}$ are in $C^{1+\alpha}(X \times$ $W,(0,1))$ and $f: X \rightarrow \mathbf{R}$ is in $C^{1+\alpha}(X, \mathbf{R})$. For a given value of $w$, let $\mu_{w}$. be the unique invariant probability measure for the system. Then $\left\langle\mu_{n}, f\right\rangle$ is a differentiable function of the parameter $w$.

Proof. For simplicity's sake we couch our argument in terms of the case where $X$ and $W$ are one-dimensional; the only modifications necessary for the general case are the substitution of operator norms for absolute values, etc.

We let $\rho$ be the metric on $X$ given by the shortest distance along paths in $X$; then we have

$$
\rho\left(\beta_{i}(x), \beta_{i}(y)\right)<\lambda \rho(x, y) .
$$

Note that $|x-y| \leq \rho(x, y)$, and thus any function which is $C^{\alpha}$ with respect to the $|\cdot|$ metric is $C^{\alpha}$ with respect to $\rho$.

From the arguments of the previous section we know that if $T$ is the linear operator from $C$ to $C$ given by:

$$
(T f)(x)=\sum_{i} p_{i}(x, w) f\left(\beta_{i}(x, w)\right),
$$


then the sequence $T^{k} f$ converges uniformly to the constant function with value $\left\langle\mu_{w}, f\right\rangle$. It is apparent that $T^{k} f$ is a differentiable function of $w$; we show that the derivatives converge uniformly, thus proving the theorem.

Let $g: X \times W \rightarrow \mathbf{R}$ and let us consider $D T g=(\partial / \partial w)(T g)$ :

$$
\begin{aligned}
\operatorname{DTg}(x, w) & =D\left(\sum_{i} p_{i}(x, w) g\left(\beta_{i}(x, w), w\right)\right) \\
& =A g+T D g,
\end{aligned}
$$

where

$$
A g(x, w)=\sum_{i}\left(D p_{i}(x, w) g\left(\beta_{i}(x, w), w\right)+p_{i}(x, w) g^{\prime}\left(\beta_{i}(x, w), w\right) D \beta_{i}(x, w)\right)
$$

Note that we use $g^{\prime}$ to denote $\partial g / \partial x$ as opposed to $D g=\partial g / \partial w$.

While we assume that $f$ is independent of $w, T^{k} f$ depends on $w$ for $k>0$. We thus have:

$$
\begin{aligned}
D T f & =A f \\
D T^{2} f & =A T f+T D T f \\
& =A T f+T A f \\
D T^{3} f & =A T^{2} f+T A T f+T^{2} A f .
\end{aligned}
$$

And in general,

$$
D T^{k} f=\sum_{j=0}^{k} T^{j} A T^{k-j} f .
$$

We now turn our attention to upper bounds for $\Gamma\left(T^{j} A T^{k-j} f\right)$.

LEMMA 3.2. Suppose $p_{i}, \beta_{i}$, and $f$ are $C^{\alpha}$ functions for some $\alpha>0$. Then there exist constants $c$ and $r \in(0,1)$, independent of $f$, so that

$$
\Gamma\left(T^{k} f\right) \leq c r^{k} \sup \{\Gamma(f), \Psi(f)\},
$$

where the operator $\Psi$ is defined by

$$
\Psi(f)=\sup _{x \neq y} \frac{|f(x)-f(y)|}{\rho(x, y)^{\alpha}} .
$$

Proof. For a function $h$ which depends on $w$ as well as $x$, we define

$$
\begin{aligned}
\Gamma(h) & =\sup _{n \in w} \sup _{x, y \in X}|h(x, w)-h(y, w)| ; \\
\Psi(h) & =\sup _{x \in w^{\prime}} \sup _{x \neq y} \frac{|h(x, w)-h(y, w)|}{\rho(x, y)^{\alpha}} .
\end{aligned}
$$

In this proof we do not show dependence on $w$ explicitly. Note that $\Psi$ is subadditive.

Let $z$ be any point in $X$. We have the following upper bound on $\Psi(T f)$ :

$$
\begin{aligned}
\frac{T f(x)-T f(y)}{\rho(x, y)^{\alpha}}= & \sum_{i}\left(\frac{p_{i}(x)-p_{i}(y)}{\rho(x, y)^{\alpha}} f\left(\beta_{i}(x)\right)+p_{i}(y) \frac{f\left(\beta_{i}(x)\right)-f\left(\beta_{i}(y)\right)}{\rho(x, y)^{\alpha}}\right) \\
= & \sum_{i} \frac{p_{i}(x)-p_{i}(y)}{\rho(x, y)^{\alpha}}\left(f\left(\beta_{i}(x)\right)-f(z)\right) \\
& +\sum_{i} p_{i}(y) \frac{f\left(\beta_{i}(x)\right)-f\left(\beta_{i}(y)\right)}{\rho\left(\beta_{i}(x), \beta_{i}(y)\right)^{\alpha}} \frac{\rho\left(\beta_{i}(x), \beta_{i}(y)\right)^{\alpha}}{\rho(x, y)^{\alpha}}
\end{aligned}
$$


where we have used the fact that $\sum p_{i} \equiv 1$. Taking the supremum over $x \neq y$ and $w \in W$, we obtain

$$
\Psi(T f) \leq \sum_{i} \Psi\left(p_{i}\right) \Gamma(f)+\lambda^{\alpha} \Psi(f)
$$

We can iterate this inequality to obtain

$$
\Psi\left(T^{k} f\right) \leq \frac{1}{1-\lambda^{\alpha}} \sum_{i} \Psi\left(p_{i}\right) \Gamma(f)+\lambda^{k \alpha} \Psi(f) .
$$

From Lemma 2.1 we have:

$$
\Gamma\left(T^{k} f\right) \leq\left(1-2 q^{k}\right) \Gamma(f)+2 q^{k} \Psi(f) \lambda^{k \alpha}\left(\operatorname{diam}_{\rho} X\right)^{\alpha},
$$

where $q$ is a lower bound for $p_{1}, \ldots, p_{n}$; we assume $q \in\left(0, \frac{1}{2}\right)$.

Thus we have

$$
\left(\begin{array}{l}
\Gamma\left(T^{k} f\right) \\
\Psi\left(T^{k} f\right)
\end{array}\right) \leq\left(\begin{array}{cc}
1-2 q^{k} & 2 q^{k} \lambda^{k \alpha}\left(\operatorname{diam}_{\rho} X\right)^{\alpha} \\
1 /\left(1-\lambda^{\alpha}\right) \sum_{i} \Psi\left(p_{i}\right) & \lambda^{k \alpha}
\end{array}\right)\left(\begin{array}{l}
\Gamma(f) \\
\Psi(f)
\end{array}\right)
$$

The $\leq$ sign here means that each component of the vector on the left is not greater than the corresponding component of the vector on the right. Let us denote the matrix in this inequality $M_{k}$, and investigate its dynamics. Its eigenvalues are given by

$$
e_{ \pm}=\left(1-2 q^{k}+\lambda^{k \alpha} \pm\left[\left(1-2 q^{k}-\lambda^{k \alpha}\right)^{2}+8 q^{k} \lambda^{k \alpha} K\right]^{1 / 2}\right) / 2
$$

where

$$
K=\frac{\left(\operatorname{diam}_{\rho} X\right)^{\alpha}}{1-\lambda^{\alpha}} \sum_{i} \Psi\left(p_{i}\right)
$$

Since $q<\frac{1}{2}$, the eigenvalue $e_{+}$has the larger absolute value. Using the fact that the graph of $\sqrt{x}$ lies below the tangent line at $x_{0}=\left(1-2 q^{k}-\lambda^{k \alpha}\right)^{2}$, we obtain

$$
\begin{aligned}
e_{+} & \leq\left(1-2 q^{k}+\lambda^{k \alpha}+1-2 q^{k}-\lambda^{k \alpha}+4 q^{k} \lambda^{k \alpha} \frac{K}{1-2 q^{k}-\lambda^{k \alpha}}\right) / 2 \\
& \leq 1-2 q^{k}\left(1-\frac{K \lambda^{k \alpha}}{1-2 q^{k}-\lambda^{k \alpha}}\right) .
\end{aligned}
$$

Since the limit of the term in parentheses is 1 as $k \rightarrow \infty$, we can choose a value $j$ for $k$ so that $\left|e_{+}\right|<1$ and the origin is a stable fixed point of $M_{j}$.

Let $E$ be a matrix whose columns are the eigenvectors of $M_{j}$; thus $P=E^{-1} M_{j} E$ is diagonal. Then $\|P\|_{\text {op }}=e_{+}$, where $\|\cdot\|_{\text {op }}$ is the operator norm derived from the supremum norm on $\mathbf{R}^{\text {? }}$. Let $u$ and $v<j$ be positive integers such that $k=u j+v$. Then

$$
\left(\begin{array}{l}
\Gamma\left(T^{k} f\right) \\
\Psi\left(T^{k} f\right)
\end{array}\right) \leq M_{i}^{v} E P^{\prime \prime} E^{-1}\left(\begin{array}{l}
\Gamma(f) \\
\Psi(f)
\end{array}\right)
$$

Thus

$$
\sup \left\{\Gamma\left(T^{k} f\right), \Psi\left(T^{k} f\right)\right\} \leq\left\|M_{1}\right\|_{\text {op }}^{c}\|E\|_{\text {op }} e_{+}^{u}\left\|E^{-1}\right\|_{\text {op }} \sup \{\Gamma(f), \Psi(f)\} .
$$

Thus the lemma holds with $c=\left\|M_{1}\right\|_{\text {op }}^{i}\|E\|_{\text {op }}\left\|E^{-1}\right\|_{\mathrm{op}} / e_{+}$and $r=e_{+}^{1 / i}$. 
Lemma 3.3. Suppose $p_{i}, \beta_{i}$, and $f$ are $C^{1+\alpha}$ functions for some $\alpha>0$. Then there exist constants $c$ and $r<1$, independent of $f$ and $w$, so that

$$
\sup \left\{\Gamma\left(T^{k} f\right),\left\|\left(T^{k} f\right)^{\prime}\right\|, \Psi\left(\left(T^{k} f\right)^{\prime}\right)\right\} \leq c r^{k} \sup \left\{\Gamma(f),\left\|f^{\prime}\right\|, \Psi\left(f^{\prime}\right)\right\}
$$

Proof. The proof of this lemma is a three-dimensional argument analogous to the two-dimensional argument used to prove Lemma 3.2 and we omit it.

Proof of Theorem 3.1 continued. Using Lemma 3.3, we choose constants $c_{1}$ and $r_{1}<1$ such that

$$
\sup \left\{\Gamma\left(T^{k} f\right),\left\|\left(T^{k} f\right)^{\prime}\right\|, \Psi\left(\left(T^{k} f\right)^{\prime}\right)\right\} \leq c_{1} r_{1}^{k} \sup \left\{\Gamma(f),\left\|f^{\prime}\right\|, \Psi\left(f^{\prime}\right)\right\} .
$$

We recall the definition (3) of the operator $A$. Since $\sum_{i} D p_{i}=0$, for arbitrary $z \in X$, we may write

$$
\begin{aligned}
A g(x, w)= & \sum_{i} D p_{i}(x, w)\left(g\left(\beta_{i}(x, w), w\right)-g(z, w)\right) \\
& +\sum_{i} p_{i}(x, w) g^{\prime}\left(\beta_{i}(x, w), w\right) D \beta_{i}(x, w)
\end{aligned}
$$

Thus

$$
\|A g\| \leq a \Gamma(g)+b\left\|g^{\prime}\right\|,
$$

where $a=\sum_{i}\left\|D p_{i}\right\|$ and $b=\sup _{i}\left\|D \beta_{i}\right\|$.

Thus

$$
\left\|A T^{k-j} f\right\| \leq(a+b) c_{1} r_{1}^{k-j} \sup \left\{\Gamma(f),\left\|f^{\prime}\right\|, \Psi\left(f^{\prime}\right)\right\} .
$$

Note that if $h^{\prime}$ exists and is bounded, then $\Psi(h) \leq\left\|h^{\prime}\right\|\left(\operatorname{diam}_{\rho} X\right)^{1-\alpha}$. Thus we may calculate

$$
\begin{aligned}
\frac{|A g(x)-A g(y)|}{\rho(x, y)^{\alpha}}= & \sum_{i} \frac{\left(D p_{i}(x)-D p_{i}(y)\right)}{\rho(x, y)^{\alpha}}\left(g\left(\beta_{i}(x)\right)-g(z)\right) \\
& +\sum_{i} D p_{i}(y) \frac{g\left(\beta_{i}(x)\right)-g\left(\beta_{i}(y)\right)}{\rho(x, y)^{\alpha}}+\sum_{i} \frac{p_{i}(x)-p_{i}(y)}{\rho(x, y)^{\alpha}} g^{\prime}\left(\beta_{i}(x)\right) D \beta_{i}(x) \\
& +\sum_{i} p_{i}(y) \frac{g^{\prime}\left(\beta_{i}(x)\right)-g^{\prime}\left(\beta_{i}(y)\right)}{\rho(x, y)^{\alpha}} D \beta_{i}(x) \\
& +\sum_{i} p_{i}(y) g^{\prime}\left(\beta_{i}(y)\right) \frac{D \beta_{i}(x)-D \beta_{i}(y)}{\rho(x, y)^{\alpha}} \\
\leq & \sum_{i} \Psi\left(D p_{i}\right) \Gamma(g)+\sum_{i}\left\|D p_{i}\right\| \Psi\left(g \circ \beta_{i}\right)+\sum_{i} \Psi\left(p_{i}\right)\left\|g^{\prime}\right\|\left\|D \beta_{i}\right\| \\
& +\sum_{i} p_{i}(y) \Psi\left(g^{\prime}\right)\left\|D \beta_{i}\right\|+\sum_{i} p_{i}(y)\left\|g^{\prime}\right\| \Psi\left(D \beta_{i}\right) \\
\leq & \sum_{i} \Psi\left(D p_{i}\right) \Gamma(g)+\sum_{i}\left\|D p_{i}\right\|\left\|g^{\prime}\right\| \lambda\left(\operatorname{diam}_{\rho} X\right)^{1-\alpha} \\
& +\sum_{i}\left\|p_{i}^{\prime}\right\|\left(\operatorname{diam}_{\rho} X\right)^{\prime-\alpha}\left\|g^{\prime}\right\|\left\|D \beta_{i}\right\| \\
& +\sup _{i}\left\|D \beta_{i}\right\| \Psi\left(g^{\prime}\right)+\sup _{i} \Psi\left(D \beta_{i}\right)\left\|g^{\prime}\right\| .
\end{aligned}
$$

Thus

$$
\Psi(A g) \leq u \Gamma(g)+v\left\|g^{\prime}\right\|+w \Psi\left(g^{\prime}\right),
$$


where

$$
u=\sum_{i} \Psi\left(D p_{i}\right), \quad w=\sup _{i}\left\|D \beta_{i}\right\|
$$

and

$$
v=\lambda\left(\operatorname{diam}_{\rho} X\right)^{1-\alpha} \sum_{i}\left\|D p_{i}\right\|+\left(\operatorname{diam}_{\rho} X\right)^{1-\alpha} \sum_{i}\left\|p_{i}^{\prime}\right\|\left\|D \beta_{i}\right\|+\sup _{i} \Psi\left(D \beta_{i}\right) .
$$

Therefore,

$$
\Gamma\left(A T^{k-j} f\right) \leq 2\left\|A T^{k-j} f\right\| \leq 2(a+b) c_{1} r_{1}^{k-j} \sup \left\{\Gamma(f),\left\|f^{\prime}\right\|, \Psi\left(f^{\prime}\right)\right\},
$$

and

$$
\Psi\left(A T^{k-j} f\right) \leq(u+v+w) c_{1} r_{1}^{k-j} \sup \left\{\Gamma(f),\left\|f^{\prime}\right\|, \Psi\left(f^{\prime}\right)\right\} .
$$

Finally, applying Lemma 3.2 to $A T^{k-j} f$, we obtain constants $c_{2}$ and $r_{2}<1$ such that

$$
\begin{aligned}
\Gamma\left(T^{j} A T^{k-j} f\right) & \leq c_{2} r_{2}^{j} \sup \left\{\Gamma\left(A T^{k-j} f\right), \Psi\left(A T^{k-j} f\right)\right\} \\
& \leq c_{2} r_{2}^{j}(2 a+2 b+u+v+w) c_{1} r_{1}^{k-j} \sup \left\{\Gamma(f),\left\|f^{\prime}\right\|, \Psi\left(f^{\prime}\right)\right\} .
\end{aligned}
$$

Then

$$
\begin{aligned}
\Gamma\left(D T^{k} f\right) & \leq \sum_{j=0}^{k} \Gamma\left(T^{j} A T^{k-j} f\right) \\
& \leq(k+1) c_{2} r_{2}^{j}(2 a+2 b+u+v+w) c_{1} r_{1}^{k-j} \sup \left\{\Gamma(f),\left\|f^{\prime}\right\|, \Psi\left(f^{\prime}\right)\right\} .
\end{aligned}
$$

It is easily shown that $\|T h-h\| \leq \Gamma(h)$. Referring to formula (2), we have

$$
\begin{aligned}
&\left\|D T^{k+1} f-D T^{k} f\right\| \\
& \leq\left\|A T^{k} f\right\|+\left\|T D T^{k} f-D T^{k} f\right\| \\
& \leq(a+b) c_{1} r_{1}^{k} \sup \left\{\Gamma(f),\left\|f^{\prime}\right\|, \Psi\left(f^{\prime}\right)\right\} \\
&+(k+1) c_{2} r_{2}^{j}(2 a+2 b+u+v+w) c_{1} r_{1}^{k-j} \sup \left\{\Gamma(f),\left\|f^{\prime}\right\|, \Psi\left(f^{\prime}\right)\right\} .
\end{aligned}
$$

This shows that the series $D T^{k} f$ converges uniformly and thus proves the theorem.

An interesting special case of this theorem occurs when the functions $\boldsymbol{\beta}_{i}$ are independent of $w$, so that the dependence of $\mu_{w}$ on $w$ arises solely from the dependence of the probabilities $p_{i}$ on $w$. In this case it can be shown that the conclusion of the theorem holds even if the functions are not differentiable functions of $x$, but merely $C^{\alpha}$ functions of $x$.

Concerning the complex case, we have the following:

THEOREM 3.4. Theorem 3.1 remains true if $X$ and $W$ are compact subsets of $C^{N}$ and $f: X \rightarrow C$.

Proof. Exactly the same methods used to prove Theorem 3.1 will serve to prove this theorem. In fact, since the uniform limit of holomorphic functions is holomorphic, a slightly modified version of this theorem could be proved much more easily than Theorem 3.1.

With regard to the hypotheses of Theorem 3.4 in the complex case, we note that if the probabilities $p_{1}, \ldots, p_{n}$ are to be real-valued functions and at the same time differentiable functions of $x$ and $w$, then they must be constant on the interior of $X \times W$. Thus $\mu$ has the invariance property $\mu \beta_{i}(E)=p_{i} \mu E$. 


\section{Invariant measures on Julia sets}

We now demonstrate how the results of the previous section may be applied to the study of invariant measures on Julia sets, such as the equilibrium measures studied by Brolin [5].

We consider a nonconstant holomorphic function $F: \mathbf{C}^{N} \times \mathbf{C} \rightarrow \mathbf{C}^{N}$. We shall sometimes suppress dependence on the parameter $w \in C$ and for $x \in C^{N}$ we write $F(x)$ for $F(x, w)$, etc.

Let $w_{0}$ be a fixed value of $w$ and $W$ be a compact neighbourhood of $w_{0}$. We suppose that for each $w$ there exists a compact set $J=J(w)$ satisfying the following conditions:

(i) There exists a compact simply connected neighbourhood $X$ of $J$ (independent of $w$ ) such that $X \subset F(X)$ and

$$
J=\bigcap_{k=0}^{\infty} F^{-k}(X) .
$$

(ii) For all $x \in J$ there exists $\lambda<1$ and $k$ such that

$$
\left\|\left(\left(F^{k}\right)^{\prime}(x)\right)^{-1}\right\|<\lambda \text {. }
$$

(iii) $F$ is topologically mixing on $J$; that is, for every open set $U$ which intersects $J$ nontrivially there exists $k$ such that $J \subset F^{k}(U)$.

(iv) There exists an integer $n$ so that $F$ is $n$-to-one on $X$.

An example of a set satisfying these conditions is given by the case where $F$ is a rational function on $\mathbf{C}$ and $\boldsymbol{J}$ is a hyperbolic, totally disconnected Julia set for $F$. We call particular attention to the case $F(x)=x^{2}-w$, for values of $w$ outside the Mandelbrot set $M$ and $J$ is the Julia set for $F$. The set $M$ is defined to contain just those values of the parameter $w$ for which the set $J$ is connected. For a fuller discussion of the characteristics of this system in terms of the parameter, see Douady and Hubbard [6].

It can be shown that under conditions (i)-(iii) the neighbourhood $X$ can be chosen so that the following more stringent condition holds:

(ii)' There exist $\lambda<1$ and $k$ (independent of $x$ ) so that for all $x \in X$,

$$
\left\|\left(\left(F^{k}\right)^{\prime}(x)\right)^{-1}\right\|<\lambda \text {. }
$$

If we consider the function $F^{k}$ rather than $F$ we can assume $k=1$ without loss of generality.

Let $\beta_{1}, \ldots, \beta_{n}$ denote the branches of the inverse of $F$ on $X$. Since $X$ is simply connected, the functions $\beta_{i}$ can be chosen to be continuous. Condition (ii) ${ }^{\prime}$ also implies that the functions $\beta_{1}, \ldots, \beta_{n}$ are contractions.

Let $p_{1}, \ldots, p_{n} \in(0,1)$ with $\sum p_{i}=1$. Then the hypotheses of Theorem 2.1 hold and for each $w \in W$ there is a unique invariant measure $\mu=\mu_{w}$ satisfying

$$
\mu_{w}\left(\beta_{i}(E)\right)=p_{i} \mu_{w} E .
$$

Moreover, the hypotheses of Theorem 3.4 are satisfied. Thus we can conclude that for any complex-valued function $f$ holomorphic on $X,\left\langle f, \mu_{w}\right\rangle$ is an analytic function of $w$. 
Withers $[11,12]$ discusses algorithms for the calculation of $(d / d w)\left(\left\langle f, \mu_{w}\right\rangle\right)$ in the one-dimensional case; these can easily be adapted to the $n$-dimensional case and provide values for the derivative in about as much time as is required for the calculation of $\left\langle f, \mu_{w}\right\rangle$ itself to the same accuracy.

In the one-dimensional case $F: \mathbf{C} \times \mathbf{C} \rightarrow \mathbf{C}$, we can also investigate the Hausdorff dimension HD $\left(\mu_{w^{*}}\right)$ of the measure $\mu_{w^{\prime}}$ as a function of $w$. We shall use the following formula for the Hausdorff dimension of a measure which is derived from its definition in Manning [9]:

$$
\operatorname{HD}(\mu)=\frac{h_{\mu}(F)}{\left\langle\log \left|F^{\prime}\right|, \mu\right\rangle},
$$

where $h_{\mu}(F)$ is the entropy of $\mu$. In the case at hand, this is given by $h_{\mu}(F)=$ $-\sum p_{i} \log p_{i}$, and thus

$$
\frac{1}{\operatorname{HD}\left(\mu_{n}\right)}=\frac{\left\langle\log \left|F^{\prime}\right|, \mu_{n}\right\rangle}{-\sum p_{i} \log p_{i}} .
$$

Since $X$ is simply connected and does not contain the critical points of $F^{\prime}, \log F^{\prime}$ can be defined to be analytic on $X$. Thus

$$
\delta(w)=\frac{\left\langle\log F^{\prime}, \mu_{n}\right\rangle}{-\sum p_{i} \log p_{i}}
$$

is an analytic function of $w$. Thus we have the following theorem:

THEOREM 4.1. Under hypotheses (i)-(iv), $\operatorname{Re} \delta(w)=1 / \mathrm{HD}\left(\mu_{w}\right)$ is a harmonic function of $w$.

It is interesting to compare this result with that obtained by Ruelle [10], who proved that when the Julia set for a rational function is hyperbolic (similar to our hypothesis (ii)) the Hausdorff dimension of the set depends real-analytically on a parameter. The Hausdorff dimension of a set is equal to the supremum of the Hausdorff dimensions of measures supported on that set.

Theorem 4.1 includes the case of the mapping $F(x)=x^{2}-w$ for values of $w$ outside $M$. The question of values of $w$ inside the Mandelbrot set was treated for general polynomials by Manning [9], who showed with $p_{i}=1 / n$ that $\operatorname{HD}(\mu)$ is always unity.

The quantity $\operatorname{Im} \delta(w)$ is intriguing. Its value depends on the choice of $X$; however, if the value of $\operatorname{Im} \delta\left(w_{0}\right)$ for a particular $w_{0}$ is known, then the values of $\operatorname{Im} \delta(w)$ for values of $w$ near $w_{0}$ are determined; thus $\operatorname{Im} \delta(w)$ is defined locally up to a constant independent of the choice of $X$. In the quadratic case $F(x)=x^{2}-w$, through eyeball examination of the sets involved, it can be determined that as $w$ travels counterclockwise once around a path enclosing $M$ the value of $\operatorname{Im} \delta(w)$ increases by $-\pi / \sum p_{i} \log p_{i}$.

\section{REFERENCES}

[1] M. Barnsley \& S. Demko. Iterated function systems and the global construction of fractals. Proc. R. Soc. Lond. A399 (1985), 243-275. 
[2] M. Barnsley, S. Demko, J. Elton \& J. Geronimo. Invariant measures for Markov processes arising from iterated function systems with place-dependent probabilities. Ann. Inst. Henri Poincaré 24 (3) (1988), 367-394.

[3] M. Barnsley \& J. Elton. Stationary attractive measures for a class of Markov chains arising from function iteration. A new class of Markov processes for image encoding. Adv. in Appl. Probab. 20 (1988), 14-32.

[4] M. Barnsley \& A. Harrington. A Mandelbrot set for pairs of linear maps. Physica 15D (1985), 421-432.

[5] H. Brolin. Invariant sets under iteration of rational functions. Ark. Mat. 6 (1965), 103-144.

[6] A. Douady \& J. Hubbard. Itération des polynômes quadratiques complexes. C.R. Acad. Sc. Paris, Sér. I 294 (1982), 123-126.

[7] J. Elton. An ergodic theorem for iterated maps. Ergod. Th. \& Dynam. Sys. 7 (1987), 481-488.

[8] S. Karlin. Some random walks arising in learning models Pac. J. Math. 3 (1953), 752-756.

[9] A. Manning. The dimension of the maximal measure for a polynomial map. Ann. Math. 119 (1984), 425-430.

[10] D. Ruelle. Repellers for real analytic maps. Ergod. Th. \& Dynam. Sys. 2 (1982), 99-107.

[11] W. Withers. Calculation of Taylor series for Julia sets in powers of a parameter, in: Chaotic Dynamics and Fractals, pp. 203-213, Academic Press: New York, 1986.

[12] W. Withers. Calculating derivatives with respect to parameters in iterated function systems. Physica 28D (1987), 206-214. 